\title{
Induction of Trabecular Meshwork Cells From Induced Pluripotent Stem Cells
}

\author{
Qiong J. Ding, Wei Zhu, Amy C. Cook, Kristin R. Anfinson, Budd A. Tucker, and Markus H. Kuehn
}

The Stephen A. Wynn Institute for Vision Research, Department of Ophthalmology and Visual Sciences, University of Iowa, Iowa City, Iowa, United States

Correspondence: Markus H. Kuehn, The University of Iowa, Department of Ophthalmology and Visual Sciences, 200 Hawkins Drive, Iowa City, IA 52242, USA;

markus-kuehn@uiowa.edu.

Submitted: May 15, 2014

Accepted: September 21, 2014

Citation: Ding QJ, Zhu W, Cook AC, Anfinson KR, Tucker BA, Kuehn MH. Induction of trabecular meshwork cells from induced pluripotent stem cells. Invest Ophthalmol Vis Sci. 2014;55:7065-7072. DOI:10.1167/ iovs. 14-14800
Purpose. Loss or dysfunction of trabecular meshwork (TM) cells has been associated with the development of pathologically elevated IOP, and it is conceivable that replacement of damaged TM cells could restore function to the TM. We propose that the use of TM-like cells derived from induced pluripotent stem cells (iPSCs) created from a patient's own dermal fibroblasts offers the best solution to this challenge. Here we demonstrate that mouse iPSCs can be induced to differentiate into TM-like cells suitable for autologous transplantation.

MethoDs. Directed induction of stem cell differentiation was achieved through coculture of mouse iPSCs with human TM cells for up to 21 days. The resultant TM-like cells (iPSC-TM) were characterized morphologically, immunohistochemically, and functionally.

REsults. The iPSC-TM cells closely resembled cultured human TM cells morphologically and began to express many markers of TM cells while ceasing to express pluripotency markers such as Nanog, Oct4, and Sox 2 . Functionally, these cells developed the ability to phagocytose particles. Finally, exposure to dexamethasone or phorbol 12-myristate acetate caused a distinct increase in the production and secretion of myocilin and matrix metalloproteinase-3, respectively, behavior characteristic of TM cells.

Concuusions. Our data demonstrate that iPSCs can be induced to assume a phenotype that resembles native TM cells in many important aspects. Not only do these cells represent a valuable research tool, but transplantation into glaucomatous eyes with elevated IOP may also restore function to the TM, resulting in re-establishment of IOP.

Keywords: induced pluripotent stem cells, trabecular meshwork, glaucoma anterior segment
G laucoma is a leading cause of irreversible blindness worldwide. ${ }^{1}$ The development of this disease is frequently accompanied by an increase in intraocular pressure (IOP) that can lead to visual field loss and blindness as a result of ganglion cell death and optic nerve atrophy. ${ }^{2-4}$

The aqueous humor inside the eye is continuously produced, and the majority of the fluid exits through the trabecular meshwork (TM). ${ }^{5}$ Trabecular meshwork outflow pathways are critical in providing resistance to aqueous humor outflow and in maintaining IOP in the healthy eye. ${ }^{6-8}$ The precise etiology of the elevated IOP in primary openangle glaucoma (POAG) is still unknown, but numerous studies point to an outflow obstruction of the intraocular fluid within the TM or the endothelial lining of Schlemm's canal.9,10 Cellularity is decreased in the TM of POAG patients, ${ }^{11-15}$ and it is likely that TM cells become dysfunctional prior to death and increasingly fail to carry out their physiological roles, leading to enhanced aqueous humor outflow resistance and the development of pathologically elevated IOP. ${ }^{16-18}$

It is conceivable that replacement of damaged or lost TM cells could restore function to the TM and re-establish IOP control. Data have shown that laser trabeculoplasty causes division of early TM cells and that these cells migrate and repopulate the burn sites. The observed increase in outflow facility and decrease in IOP in POAG patients may be due to the activity of these newly created cells either through direct interaction with the extracellular matrix of the TM or through a paracrine effect on the remaining endogenous cells. ${ }^{19-21}$ Alternatively, it may be possible to achieve the same effect using an endogenously supplied cell population. The use of stem or progenitor cells for the purpose of cellular replacement is promising because of their demonstrated ability to induce tissue reconstruction and functional regeneration. ${ }^{22,23}$

Pluripotent cells can be obtained from many tissues of early embryos or adults. Several groups have successfully isolated TM progenitor cell populations from free-floating neurospheres derived from human TM cells, TM insert cells, or human TM as side populations or clones. ${ }^{24-26}$ Cells derived through these approaches closely resemble primary TM cells, but harvesting tissue to obtain a sufficient number of such cells is not feasible from a living eye. In order to develop transplantation of TM cells as a clinically viable treatment approach for POAG, we believe that harvesting of donor cells must be reliable, must be minimally invasive, and must yield a significant number of cells. Furthermore, autologous transfer to avoid immune responses and ethical concerns associated with the use of embryonic stem cells would be desirable. One approach that can overcome these hurdles is the use of induced pluripotent stem cells (iPSCs). Induced PSCs are generated via genetic reprogramming of adult somatic cells to pluripotency through simultaneous expression of four transcription factors: Oct4, Sox2, Klf4, 
and c-Myc. ${ }^{27}$ Ultimately, the use of TM-like cells derived from iPSCs created from a patient's own dermal fibroblasts may offer the best solution to the challenge of TM cell replacement therapy. Here, we demonstrate that mouse iPSCs derived from dermal fibroblasts can be induced to differentiate into TM-like cells, designated iPSC-TM, and demonstrate that these cells are similar morphologically and functionally to TM cells.

\section{MeTHODS}

\section{Primary TM Cell Culture}

Human eyes were procured through the Iowa Lions Eye Bank with the next of kin consent and processed within 8 hours post mortem. The anterior chamber was excised from the globe, and TM tissue was removed using a blunt dissection technique in which a $0.5-\mathrm{mm}$ curette was used to separate a continuous strand of TM tissue away from the scleral spur, ciliary muscle, and Schwalbe's line. Trabecular meshwork cells were isolated from the tissue using collagenase digestion and cultured in "human complete medium" as reported by Stamer et al. ${ }^{28}$ The tissue was rinsed twice with Dulbecco's modified Eagle's medium (DMEM) and digested for 2 hours at $37^{\circ} \mathrm{C}$ in Dulbecco's phosphate-buffered saline (cation free, $\mathrm{pH} 7.4$ ) containing 4 $\mathrm{mg} / \mathrm{mL}$ collagenase-A (Worthington Biochemical Corp., Freehold, NJ, USA) and $4 \mathrm{mg} / \mathrm{mL}$ human serum albumin (Abcam, Cambridge, MA, USA). Following digestion, the cells were cultured in "human complete medium" containing 199E medium (Gibco, Grand Island, NY, USA), 20\% fetal bovine serum (Gibco), $90 \mu \mathrm{g} / \mathrm{mL}$ porcine heparin (SigmaAldrich Corp., St. Louis, MO, USA), 20 U/mL Endothelial Cell Growth Supplement (Sigma-Aldrich Corp.), and $1.7 \mathrm{mM} \mathrm{L-}$ glutamine (Sigma-Aldrich Corp.) in six-well culture plates coated with $1 \%$ gelatin (Sigma-Aldrich Corp.). Cultures were maintained at $37^{\circ} \mathrm{C}$ in $5 \% \mathrm{CO}_{2}$ in a humidified tissue culture incubator.

\section{Preparation of iPSCs}

Fibroblasts were cultured from 4- to 6-week-old transgenic mice constitutively expressing the reporter gene dsRed (B6.Cg$\operatorname{Tg}$ (ACTB-DsRed*MST)1Nagy/J (Jackson Laboratory, Bar Harbor, ME, USA). Induced PSCs were generated from dermal fibroblasts by lentiviral transduction of the four transcription factors Oct4, Sox2, Klf4, and c-Myc as previously described. ${ }^{29}$ Five days after infection, cells were passaged onto a monolayer of inactive mouse embryonic fibroblasts and fed daily with pluripotency media containing DMEM F-12 medium (Gibco), 15\% heat-inactivated fetal bovine serum (FBS; Lifeblood Medical, Inc., Freehold, NJ, USA), 0.0008\% $\beta$-mercaptoethanol (Sigma-Aldrich Corp.), $1 \% 100 \times$ nonessential amino acids (Gibco), $1 \times 10^{6}$ units/L leukemia inhibitory factor (LIF/ ESGRO; Millipore, Billerica, MA, USA), and 1\% Primocin (Gibco). At 3 to 5 weeks post infection, iPSC colonies were picked, passaged, and clonally expanded for additional experimentation.

\section{Differentiation of iPSCs}

Mouse iPSCs were passaged onto feeder-free tissue culture plates coated with Matrigel (BD Biosciences, San Jose, CA, USA) and expanded in pluripotency media for 3 days prior to differentiation. Cells were then cocultured for up to 21 days beneath immortalized human TM cells (hTM5; a gift of Abe Clark ${ }^{30}$ ) that were plated in cell culture inserts (Corning Inc., Corning, NY, USA). Cultures were fed daily with fresh media containing MEM-alpha (Gibco), 10\% FBS (Gibco), and 1\% Primocin (Gibco).

\section{Immunohistochemistry}

Induced PSC-TM were cultured on coverslips in six-well plates and harvested after 7, 14, or 21 days (D7, D14, and D21, respectively). Cells were rinsed with PBS and fixed in $4 \%$ paraformaldehyde for 20 minutes at room temperature. Sections were rinsed again in PBS, blocked with $1 \%$ bovine serum albumin (BSA), incubated with the primary antibody, and finally incubated with the corresponding secondary antibody. All sections were treated with 4',6-diamidino-2phenylindole (DAPI) to facilitate orientation. Primary antibodies used were anti-caveolin 1 (Abcam), collagen 4 alpha 5 chain (Santa Cruz Biotechnology, Dallas, TX, USA), matrix protein Gla (Santa Cruz Biotechnology), myocilin (Abcam), tissue inhibitor of matrix metalloproteinase-3 (Thermo Pierce, Rockford, IL, USA), Vcam1 (R\&D Systems, Minneapolis, MN, USA), Oct4 (Santa Cruz Biotechnology), Sox2 (R\&D Systems), and Nanog (Abcam).

Secondary antibodies included donkey anti-rabbit Alexa 488, chicken anti-goat Alexa 488, and donkey anti-mouse Alexa 488 (Invitrogen, Carlsbad, CA, USA).

\section{Phagocytosis Assay}

Induced PSC-TM cells were grown in 12-well cell culture plates as described above and incubated with $E$. coliconjugated pHrodo green particles $(0.5 \mathrm{mg} / \mathrm{well}$; Invitrogen). Cells were investigated using epifluorescence microscopy. Fluorescence as a measure of ingested particles was measured using a Victor X3 multi plate reader (PerkinElmer, Waltham, MA, USA) every hour for up to 6 hours. Each data point represents the average of three independent experiments.

\section{Proteomic Analyses}

Induced PSC-TM, after removal of the inserts containing hTM5 cells, were exposed to dexamethasone (Dex, 100 nmol; Sigma-Aldrich Corp.) or phorbol 12-myristate acetate (TPA; $25 \mathrm{ng} / \mathrm{mL}$; Sigma-Aldrich Corp.) for 72 hours. The cell culture media from untreated and treated cells were collected and albumin was removed using an albumin removal kit (Thermo Pierce). Protein concentrations were determined by measuring absorbance at $280 \mathrm{~nm}$. Total protein $(50 \mu \mathrm{g})$ was loaded on TGX $4-20 \%$ gels (Bio-Rad, Hercules, CA, USA). Proteins were transferred to polyvinylidene difluoride membrane at $100 \mathrm{~V}$ for 1 hour, blocked with 5\% BSA at room temperature for 1 hour, and incubated with primary antibodies directed against either myocilin (Abcam) for Dex-treated cells or matrix metalloproteinase-3 (MMP3; Abcam) for TPA-treated cells. Immunoreactive bands were visualized using a horseradish peroxidase-conjugated secondary antibody and chemiluminescent detection. Images were captured on a Versadoc (Bio-Rad) imaging system, and band intensity was determined. Data were normalized to the detected level of tubulin content, and the ratio of myocilin and MMP3 before and after treatment was calculated. Each data point represents the results of three independent experiments.

\section{Results}

Fibroblasts isolated from red fluorescent mice were harvested (Fig. 1A), expanded, and transduced at a multiplicity of 

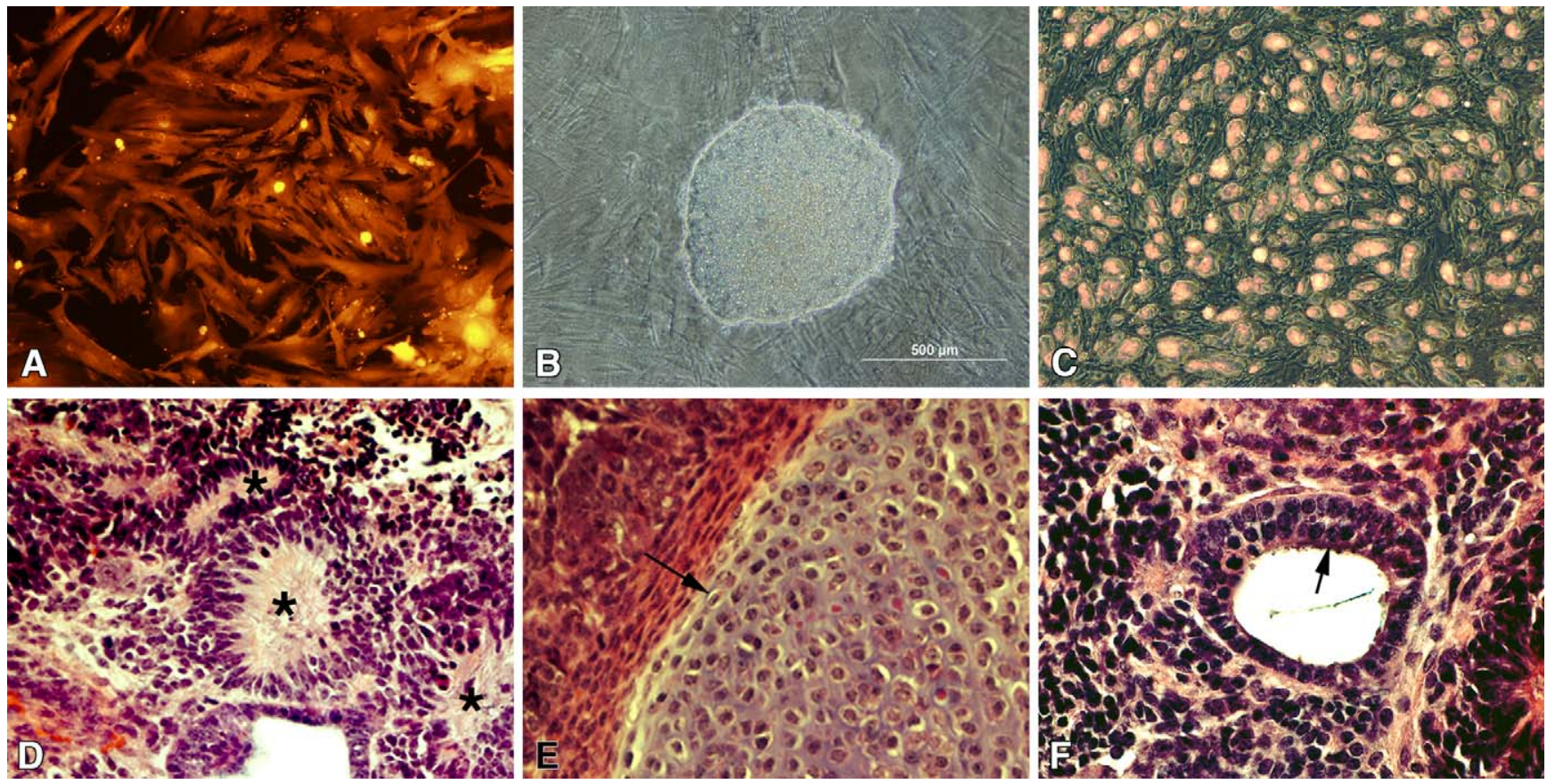

Figure 1. Microscopic analysis of fibroblasts (A), iPSC colony (B), and expanded iPSCs (C) obtained from red fluorescent mice. Histological analysis of iPSC-derived teratomas for production of cells/tissues specific to ectodermal ([D] neural rosettes, asterisk), mesodermal ([E] cartilage, arrow), and endodermal ([F] glandular epithelium, arrow) germ layers.

infection (MOI) of 5 with a lentiviral vector driving expression of the transcription factors Oct4, Sox2, Klf4, and c-Myc. At 3 to 5 weeks post transduction, iPSC colonies large enough for manual isolation (Fig. 1B) were picked and clonally expanded (Fig. 1C). To demonstrate pluripotency, $2.5 \times 10^{6}$ undifferentiated cells were injected into immunecompromised mice. Four weeks post transplantation, tumors were resected, fixed, and sectioned. Histological analysis revealed teratomas containing tissue specific to each of the three embryonic germ layers, including ectoderm-derived neural rosettes (Fig. 1D, arrow), mesoderm-derived bone (Fig. 1E, arrow), and endoderm-derived glandular epithelium (Fig. 1F, arrow)

In order to induce iPSCs to differentiate into TM-like cells, iPSCs were cocultured with the human cell line hTM $5^{30}$ for up to 21 days. This immortalized cell line was used here since it grows at a very consistent rate and displays minimal variation between passages. After approximately 7 days in coculture (D7), iPSCs started to differentiate and cells began to exhibit clear morphologic changes. Undifferentiated iPSC and TM cells were clearly distinguishable: iPSCs grew in colonies and were small round cells with small nuclei and little cytoplasm (asterisk in Figs. 2B, 2E), while primary TM cells were more elongated and spindlelike and tended to grow apart from one another until confluency was reached (Fig. 2C). Following 1 week in coculture, an increasing number of cells could be observed that had moved away from the original iPSC colonies and had assumed a cellular shape similar to that of primary TM cells (Fig. 2B). However, initially, iPSC-TM representing intermediate stages could be frequently observed, for example, those possessing smaller nuclei than fully differentiated TM cells. Interestingly, the appearance of iPSC-TM was more reminiscent of primary TM cells than the hTM5 that served to induce their differentiation (Fig. 2A). Upon reaching confluency, the morphology of primary TM cells changed, and cells became more uniformly shaped (Fig. 2F).
This appearance was also displayed by iPSC-TM cultures that reached confluency (Fig. 2E). Furthermore, confluent iPSCTM did not begin to form whorls or begin to grow in multiple layers, a behavior often observed with fibroblasts in culture.

To determine the ability of iPSC-TM to express specific TM proteins, we carried out immunohistochemistry using a series of antibodies directed against TM markers: caveolin (Cav1), ${ }^{31}$ collagen IV (Col4A5), ${ }^{32,33}$ matrix Gla protein (MGP), ${ }^{34,35}$ myocilin (MYOC), ${ }^{36}$ tissue inhibitor of metalloproteinases (TIMP3), ${ }^{26,37}$ and vascular cell adhesion molecule 1 (Vcam1). ${ }^{38}$ We also carried out immunohistochemical analysis of markers of pluripotency, such as Nanog, Oct4, and Sox $2 .{ }^{29}$ Although differences in the rate of differentiation, cell growth, and nuclear expansion existed between individual cells, the immunofluorescent analysis demonstrates that many iPSC-TM began to express TM markers 7 days after the beginning of coculture (D7). All of the tested proteins were detectable after 14 or 21 days in coculture (D14 or D21, respectively) (Fig. 3). In contrast, while initially all cells were positive for pluripotency markers, the fraction of positive cells decreased sharply over the culture period, although expression of these markers persisted in a fraction of cells until the end of the study period at D21. The labeling patterns resemble those observed in primary TM cells (Fig. A1, Appendix).

Functionally, the ability to phagocytose and degrade material is an important feature of TM cells ${ }^{39-42}$ that is not observed in iPSCs. To further assess the development of a TM cell-like phenotype, we tested the ability of iPSC-TM to carry out phagocytosis after 7,14 , and 21 days in coculture. These data were compared to those derived from undifferentiated iPSCs. Here we used pHrodo bioparticles to measure phagocytosis activity in cell culture. The fluorescence of the particles increases dramatically at acidic $\mathrm{pH}$. Under the conditions employed here, fluorescence is specifically emitted by particles that have been phagocytosed and transported to 

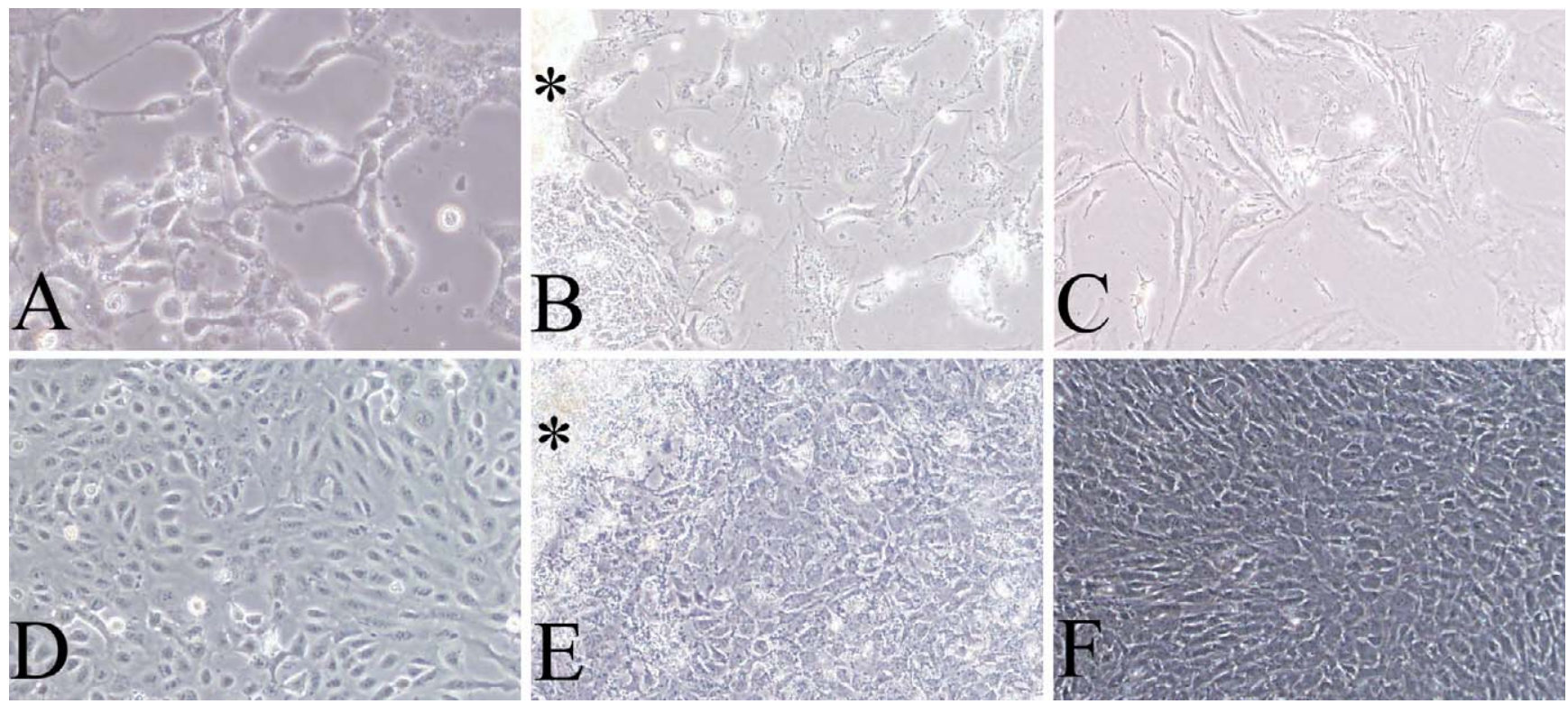

Figure 2. Morphological appearance of hTM5 cells (A, D), iPSC-TM (B, E), and primary human TM cells (C, F). Both dividing (A-C) and confluent (D-F) iPSC-TM strongly resemble primary TM cells. Some iPSC colonies persist within iPSC-TM cultures (asterisks).

acidic intracellular compartments. As shown in Figure 4A, iPSCs differentiated for 10 days engulfed the particles, and intracellular fluorescence was readily observed while particles remaining outside the cells remained nonfluorescent.

The phagocytic activity of iPSC-TM can be quantitated in a cell culture system using a fluorescence-capable microplate reader. We measured developing fluorescence hourly for up to 6 hours after addition of the pHrodo bioparticles (Fig. 4B). Undifferentiated iPSCs displayed low fluorescence throughout the entire period, indicating a very limited ability to carry out phagocytosis. However, coculture with TM cells for as few as 7 days significantly increased the ability of developing iPSC-TM to carry out this task ( $P=0.003$ after 2 hours of incubation). Overall phagocytic ability of the iPSC-TM further significantly increased after 14 days in coculture (D7 versus D1 4 at 2 hours, $P=0.22$ ). Longer coculture only modestly enhanced the ability of iPSC-TM to take up the bioparticles, and the increase was no longer statistically significant when compared to earlier time points (D14 versus D21 at 2 hours, $P=0.18$ ). At all stages of differentiation, maximal readings were achieved 2 hours after addition of the bioparticles.

As an additional functional measure, we tested the ability of iPSC-TM to secret MMP3 or MYOC when treated with TPA or Dex, respectively. Both the TPA-induced formation of matrix metalloproteinases ${ }^{43,44}$ and the increased synthesis and secretion of MYOC in response to Dex ${ }^{45-48}$ are characteristic of TM cells, but are not observed in iPSCs. Western blot analysis of iPSC-TM conditioned media (Fig. 5), collected after 3 days of exposure to either TPA or Dex, demonstrated a marked increase of MMP3 and MYOC similar to that observed in hTM5. In contrast, undifferentiated iPSCs displayed no changes in secretion of MMP3 or MYOC with or without treatment. These data demonstrate that iPSC-TM not only synthesize but also secrete both molecules at levels comparable to those observed in hTM5 cells.

\section{Discussion}

Trabecular meshwork cells fulfill a vital role in retaining the structural integrity of the TM and in maintaining healthy levels of intraocular pressure. Loss and dysfunction of these cells are likely to contribute to the development of elevated IOP in POAG. In order to further study this cell type, and as a potential avenue toward cell-based glaucoma therapy, we sought to generate TM-like cells from iPSCs derived from fibroblasts. Herein, we present data to demonstrate that iPSCs can indeed be induced to assume a phenotype that resembles native TM cells in many important aspects.

Induced PSCs were generated using adult dsRed mouse dermal fibroblasts via retroviral induction of the transcription factors Oct4, Sox2, Klf4, and c-Myc as described previously. ${ }^{29}$ These undifferentiated cells were clearly iPSCs as shown by a number of accepted criteria such as morphology and the ability to form teratomas.

Unfortunately, fewer specific markers and characteristic functional traits have been described that unequivocally identify TM cells. However, our approach to coculture iPSC with hTM5 cells induces clear morphological changes, and the resulting iPSC-TM resemble primary TM cells as reported by others. ${ }^{28,49}$ Actively dividing iPSC-TM assume an elongated spindle-like shape, but adopt a more compact shape once they have reached confluency. Remarkably, these cells resemble the morphology of primary cultured TM cells more closely than that of the hTM5 cells that serve to drive their differentiation. The reasons for this are unclear, but it is conceivable that the morphology of hTM5 is affected by immortalization and that this trait is not transmitted to newly generated iPSC-TM.

While a TM cell-specific marker has yet to be described, these cells do express a number of molecules that distinguish them from iPSCs and that, together, provide support that the derived iPSC-TM are very similar to cultured TM cells. Our data indicate that TM markers associated with endocytosis (e.g., Cav1 ${ }^{31}$ ) and extracellular matrix synthesis and degradation (e.g., Col4A5, ${ }^{32,33}$ MGP, ${ }^{34,35}$ MYOC, ${ }^{36}$ TIMP3, ${ }^{26,37}$ Vcam1 $^{38}$ ) become detectable as iPSCs differentiate into iPSC-TM while expression of pluripotency markers ceases.

Trabecular meshwork cells are known to be highly phagocytic, and it is thought that this ability plays an important role in the normal function of the conventional 


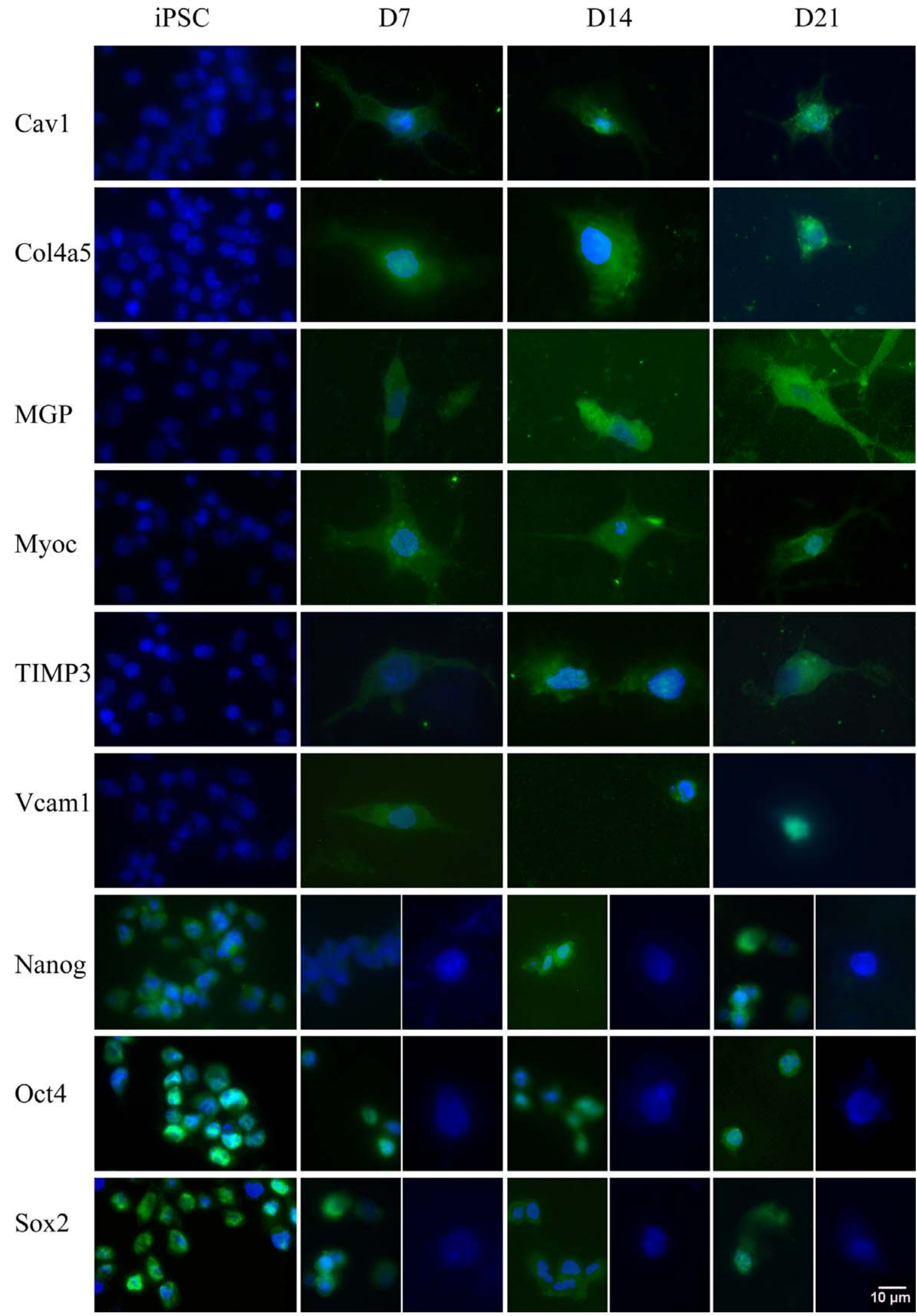

FigURE 3. Immunohistochemical detection of TM cell and pluripotency markers in iPSC-TM. Induced PSC were examined after 7, 14 , or 21 days of induction (D7, D14, or D21, respectively) and compared to uninduced pluripotent stem cells (iPSC). TM markers evaluated included caveolin 1 (Cav1), collagen 4 alpha 5 (Col4A5), matrix protein Gla (MGP), myocilin (MYOC), tissue inhibitor of metalloproteinase 3 (TIMP3), and vascular cell adhesion protein 1 (Vcam1). Markers of pluripotency included Nanog, octamer-binding transcription factor 4 (Oct4), and Sox2. Images for pluripotency markers in iPSC-TM cultures are split to demonstrate the persistence of cells resembling iPSC (left) along with iPSC-TM (right) that have ceased expression of the pluripotency markers. All images were taken at the same magnification. 

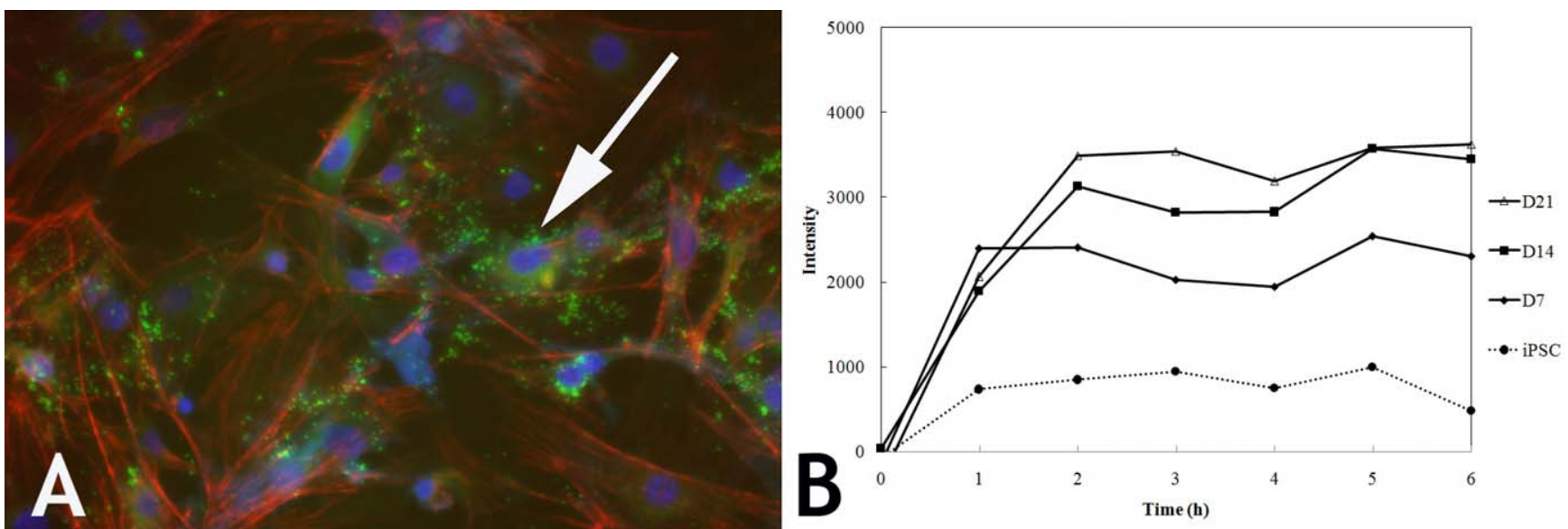

FIGURE 4. Phagocytosis in iPSC-TM. (A) Photomicrograph of iPSC-TM 2 hours after exposure to pHrodo bioparticles. Nonphagocytosed particles were not visible whereas phagocytosed particles fluoresced green (arrow). Cells counterstained with phalloidin (red) and DAPI (blue) to facilitate orientation. (B) Quantitation of phagocytic activity. The measured intensity was proportional to the amount of ingested material. Undifferentiated iPSC (broken line) exhibited little activity, whereas coculture with hTM5 cells for 7, 14, and 21 days (D7, D14, and D21, respectively) increasingly conferred the ability to phagocytose.

aqueous humor outflow pathway. ${ }^{39-42}$ The use of $\mathrm{pH}-$ sensitive particles makes it possible to directly test phagocytotic function of iPSC-TM in comparison to iPSCs or hTM5. Our data demonstrate that iPSC-TM rapidly develop the ability to phagocytose material, although additional gains in phagocytic function are detected as iPSC-TM further mature. This increase either could be due to a larger capacity of each cell to take up material or may be the result of additional cells acquiring the ability to phagocytose. This question cannot be resolved using the current experimental design, but will be addressed in future studies.

Finally, we demonstrate that iPSC-TM respond to exposure to TPA and Dex with enhanced expression of MMP3 and MYOC, respectively, similar to the responses of primary TM cells described by others. ${ }^{43-48}$ While the significance of enhanced MYOC expression remains a matter of debate, MMP3 expression likely relates to turnover and maintenance of the extracellular matrix compartment of the TM and may have important ramifications for TM pliability.

Taken together, our data demonstrate that iPSCs can be induced to assume a phenotype resembling that of native TM cells in many important aspects, morphologically and functionally. This process enables the establishment of iPSCTM lines not only from mice but also from any consenting patient. Thus not only do iPSC-TM represent a valuable research tool, but also autologous transplantation into glaucomatous eyes with elevated IOP may ultimately help to restore function to the TM. This process could help to restore normal humor outflow, and thus it might be possible to permanently reinstate IOP control.
iPSC hTM5 D7 D14 D21
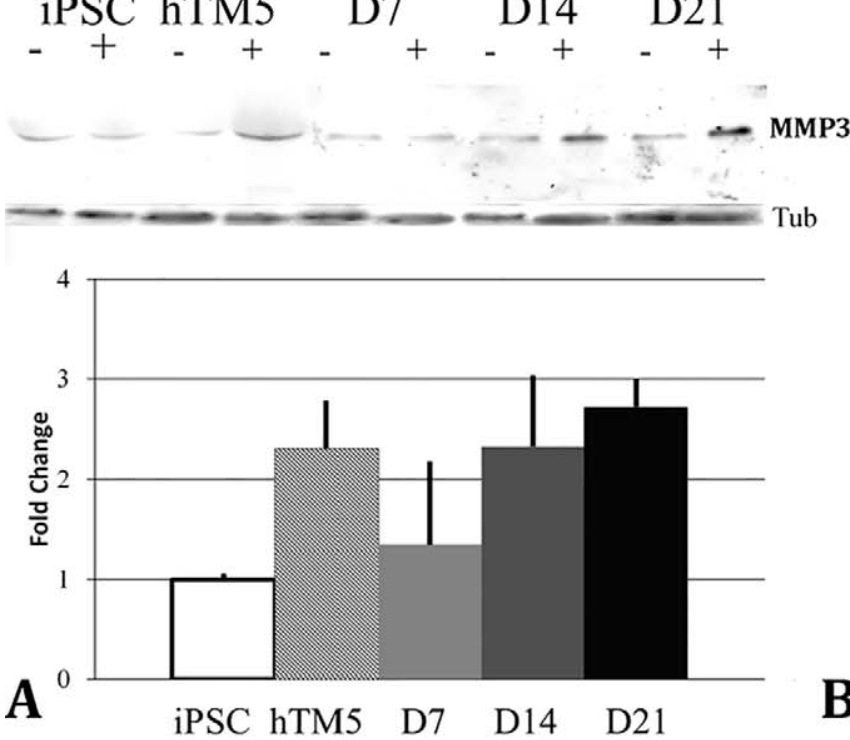

iPSC hTM5 D7 D14 D21

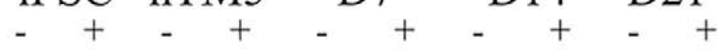

FIgURE 5. Induction of MMP3 (A) and MYOC (B) synthesis by iPSC-TM following exposure to TPA or Dex, respectively. Western blot analysis of conditioned media with $(+)$ and without $(-)$ TPA or Dex. Cells were induced for 7, 14, or 21 days (D7, D14, and D21, respectively). Values represent ratios observed between induced and uninduced cells. Uninduced iPSC and hTM5 cells are shown for comparison $(n=3)$. 


\section{Acknowledgments}

Supported by National Institutes of Health Grant R01EY022044 (MHK) and the Research to Prevent Blindness Sybil B. Harrington Special Scholar Award (MHK).

Disclosure: Q.J. Ding, None; W. Zhu, None; A.C. Cook, None; K.R. Anfinson, None; B.A. Tucker, None; M.H. Kuehn, None

\section{References}

1. Quigley HA, Broman AT. The number of people with glaucoma worldwide in 2010 and 2020. Br J Ophthalmol. 2006;90:262267.

2. Kwon YH, Fingert JH, Kuehn MH, Alward WL. Primary openangle glaucoma. $N$ Engl J Med. 2009;360:1113-1124.

3. Kuehn MH, Fingert JH, Kwon YH. Retinal ganglion cell death in glaucoma: mechanisms and neuroprotective strategies. Ophthalmol Clin North Am. 2005;18:383-395, vi.

4. Heijl A, Leske MC, Bengtsson B, et al. Reduction of intraocular pressure and glaucoma progression: results from the Early Manifest Glaucoma Trial. Arch Ophthalmol. 2002;120:12681279.

5. Goel M, Picciani RG, Lee RK, Bhattacharya SK. Aqueous humor dynamics: a review. Open Ophthalmol J. 2010;4:52-59.

6. Tamm ER. The trabecular meshwork outflow pathways: structural and functional aspects. Exp Eye Res. 2009;88:648655.

7. Keller KE, Aga M, Bradley JM, Kelley MJ, Acott TS. Extracellular matrix turnover and outflow resistance. Exp Eye Res. 2009;88: 676-682.

8. Acott TS, Kelley MJ. Extracellular matrix in the trabecular meshwork. Exp Eye Res. 2008;86:543-561.

9. Moses RA. The effect of intraocular pressure on resistance to outflow. Surv Ophthalmol. 1977;22:88-100.

10. Tamm ER, Fuchshofer R. What increases outflow resistance in primary open-angle glaucoma? Surv Ophthalmol. 2007; 52(suppl 2):S101-S104.

11. Alvarado J, Murphy C, Juster R. Trabecular meshwork cellularity in primary open-angle glaucoma and nonglaucomatous normals. Ophthalmology. 1984;91:564-579.

12. Liton PB, Challa P, Stinnett S, Luna C, Epstein DL, Gonzalez P. Cellular senescence in the glaucomatous outflow pathway. Exp Gerontol. 2005;40:745-748.

13. Welge-Lussen $\mathrm{U}$, Birke $\mathrm{K}$. Oxidative stress in the trabecular meshwork of POAG [in German]. Klin Monbl Augenbeilkd. 2010;227:99-107.

14. Tan JC, Peters DM, Kaufman PL. Recent developments in understanding the pathophysiology of elevated intraocular pressure. Curr Opin Ophthalmol. 2006;17:168-174.

15. Gabelt BT, Kaufman PL. Changes in aqueous humor dynamics with age and glaucoma. Prog Retin Eye Res. 2005;24:612-637.

16. Galdos $\mathrm{M}$, Vecino E. Ultrastructural changes in the trabecular meshwork and increased IOP. Which came first, the chicken or the egg? [in Spanish]. Arch Soc Esp Oftalmol. 2011;86:241242.

17. Singh K, Shrivastava A. Intraocular pressure fluctuations: how much do they matter? Curr Opin Opbthalmol. 2009; 20:84-87.

18. Tektas OY, Lutjen-Drecoll E. Structural changes of the trabecular meshwork in different kinds of glaucoma. Exp Eye Res. 2009;88:769-775.

19. Acott TS, Samples JR, Bradley JM, Bacon DR, Bylsma SS, Van Buskirk EM. Trabecular repopulation by anterior trabecular meshwork cells after laser trabeculoplasty. Am J Ophthalmol. 1989;107:1-6.
20. Van Buskirk EM, Pond V, Rosenquist RC, Acott TS. Argon laser trabeculoplasty. Studies of mechanism of action. Ophthalmology. 1984;91:1005-1010.

21. Bylsma SS, Samples JR, Acott TS, Van Buskirk EM. Trabecular cell division after argon laser trabeculoplasty. Arch Ophthalmol. 1988;106:544-547.

22. Weissman IL. Translating stem and progenitor cell biology to the clinic: barriers and opportunities. Science. 2000;287: 1442-1446.

23. Marchetti V, Krohne TU, Friedlander DF, Friedlander M. Stemming vision loss with stem cells. J Clin Invest. 2010; 120:3012-3021.

24. Gonzalez P, Epstein DL, Luna C, Liton PB. Characterization of free-floating spheres from human trabecular meshwork (HTM) cell culture in vitro. Exp Eye Res. 2006;82:959-967.

25. Kelley MJ, Rose AY, Keller KE, Hessle H, Samples JR, Acott TS. Stem cells in the trabecular meshwork: present and future promises. Exp Eye Res. 2009;88:747-751.

26. Du Y, Roh DS, Mann MM, Funderburgh ML, Funderburgh JL, Schuman JS. Multipotent stem cells from trabecular meshwork become phagocytic TM cells. Invest Ophthalmol Vis Sci. 2012; 53:1566-1575.

27. Takahashi K, Yamanaka S. Induction of pluripotent stem cells from mouse embryonic and adult fibroblast cultures by defined factors. Cell. 2006;126:663-676.

28. Stamer WD, Seftor RE, Williams SK, Samaha HA, Snyder RW. Isolation and culture of human trabecular meshwork cells by extracellular matrix digestion. Curr Eye Res. 1995;14:611617.

29. Tucker BA, Park IH, Qi SD, et al. Transplantation of adult mouse iPS cell-derived photoreceptor precursors restores retinal structure and function in degenerative mice. PLoS One. 2011;6:e18992.

30. Pang IH, Shade DL, Clark AF, Steely HT, DeSantis L. Preliminary characterization of a transformed cell strain derived from human trabecular meshwork. Curr Eye Res. 1994;13:51-63.

31. Kuehn MH, Wang K, Roos B, et al. Chromosome 7q31 POAG locus: ocular expression of caveolins and lack of association with POAG in a US cohort. Mol Vis. 2011;17:430-435.

32. Mao W, Liu Y, Mody A, Montecchi-Palmer M, Wordinger RJ, Clark AF. Characterization of a spontaneously immortalized bovine trabecular meshwork cell line. Exp Eye Res. 2012;105: 53-59.

33. Hernandez MR, Weinstein BI, Schwartz J, Ritch R, Gordon GG, Southren AL. Human trabecular meshwork cells in culture: morphology and extracellular matrix components. Invest Ophthalmol Vis Sci. 1987;28:1655-1660.

34. Xue W, Comes N, Borras T. Presence of an established calcification marker in trabecular meshwork tissue of glaucoma donors. Invest Ophthalmol Vis Sci. 2007;48:3184-3194.

35. Vittitow J, Borras T. Genes expressed in the human trabecular meshwork during pressure-induced homeostatic response. $J$ Cell Physiol. 2004;201:126-137.

36. Ueda J, Wentz-Hunter KK, Cheng EL, Fukuchi T, Abe H, Yue BY. Ultrastructural localization of myocilin in human trabecular meshwork cells and tissues. J Histochem Cytochem. 2000; 48:1321-1330.

37. Ronkko S, Rekonen P, Kaarniranta K, Puustjarvi T, Terasvirta $\mathrm{M}$, Uusitalo $\mathrm{H}$. Matrix metalloproteinases and their inhibitors in the chamber angle of normal eyes and patients with primary open-angle glaucoma and exfoliation glaucoma. Graefes Arch Clin Exp Ophthalmol. 2007;245:697-704.

38. Perkumas KM, Stamer WD. Protein markers and differentiation in culture for Schlemm's canal endothelial cells. Exp Eye Res. 2012;96:82-87. 


\section{APPENDiX}

39. Buller C, Johnson DH, Tschumper RC. Human trabecular meshwork phagocytosis. Observations in an organ culture system. Invest Ophthalmol Vis Sci. 1990;31:2156-2163.

40. Zhang X, Ognibene CM, Clark AF, Yorio T. Dexamethasone inhibition of trabecular meshwork cell phagocytosis and its modulation by glucocorticoid receptor beta. Exp Eye Res. 2007;84:275-284.

41. Bill A. Editorial: the drainage of aqueous humor. Invest Ophthalmol. 1975;14:1-3.

42. Johnson DH, Richardson TM, Epstein DL. Trabecular meshwork recovery after phagocytic challenge. Curr Eye Res. 1989; 8:1121-1130.

43. Alexander JP, Samples JR, Acott TS. Growth factor and cytokine modulation of trabecular meshwork matrix metalloproteinase and TIMP expression. Curr Eye Res. 1998;17: 276-285.

44. Alexander JP, Samples JR, Van Buskirk EM, Acott TS. Expression of matrix metalloproteinases and inhibitor by human trabecular meshwork. Invest Ophthalmol Vis Sci. 1991;32:172-180.

45. Clark AF, Steely HT, Dickerson JE Jr, et al. Glucocorticoid induction of the glaucoma gene MYOC in human and monkey trabecular meshwork cells and tissues. Invest Ophthalmol Vis Sci. 2001;42:1769-1780.

46. Clark AF, Wordinger RJ. The role of steroids in outflow resistance. Exp Eye Res. 2009;88:752-759.

47. Lo WR, Rowlette LL, Caballero M, Yang P, Hernandez MR, Borras T. Tissue differential microarray analysis of dexamethasone induction reveals potential mechanisms of steroid glaucoma. Invest Ophthalmol Vis Sci. 2003;44:473-485.

48. Stamer WD, Roberts BC, Howell DN, Epstein DL. Isolation, culture, and characterization of endothelial cells from Schlemm's canal. Invest Ophthalmol Vis Sci. 1998;39:1804-1812.

49. Mao W, Liu Y, Wordinger RJ, Clark AF. A magnetic bead-based method for mouse trabecular meshwork cell isolation. Invest Ophthalmol Vis Sci. 2013;54:3600-3606.
PTM

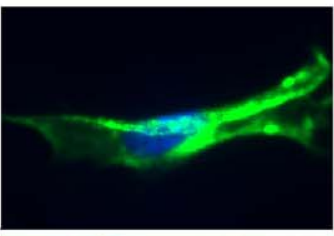

Col4a5

MGP

Myoc

TIMP3

VCam1
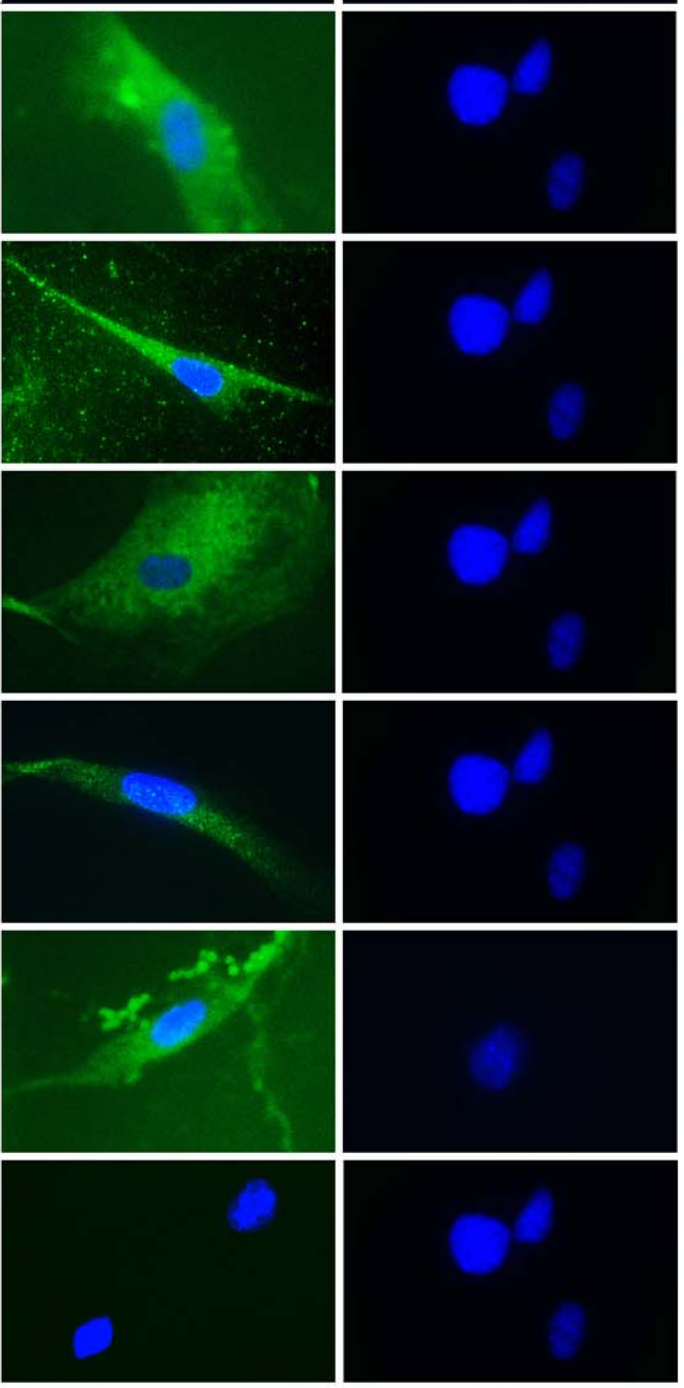

Nanog

Oct4
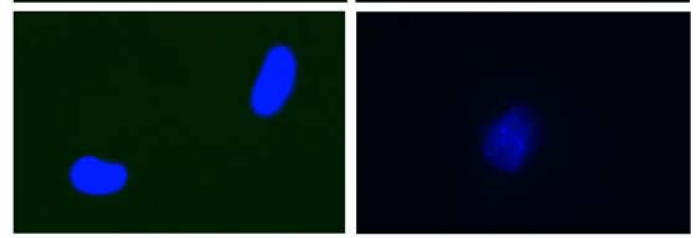

Sox 2
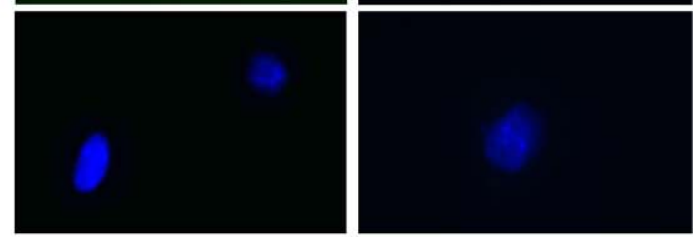

Figure A1. Immunohistochemical detection of TM cell markers in primary TM cell culture (left). The primary antibody was omitted in the images to the right as a negative control. 